\title{
Modified Spin-coating Technique to Achieve Directional Colloidal Crystallization
}

\author{
Andrew P. Bartlett, ${ }^{\#, \dagger}$ Moorthi Pichumani, ${ }^{\mathbb{I}, \ddagger}$ Maximiliano Giuliani, $\square, \ddagger$ Wenceslao González-Viñas, ${ }^{\ddagger}$ \\ and Anand Yethiraj ${ }^{*}, \dagger$
}

${ }^{\dagger}$ Department of Physics and Physical Oceanography, Memorial University, St. John’s, Newfoundland, Canada A1B 3X7

${ }^{\ddagger}$ Department of Physics and Applied Mathematics, University of Navarra, Pamplona, Spain

\section{Supporting Information}

ABSTRACT: Fabricating large single crystals with colloidal spheres as building blocks is challenging and of competitive interest. Spin-coating of colloids offers a robust technique, which is highly reproducible in obtaining colloidal crystals even at fast dynamical regimes; however, these crystals are intrinsically polycrystalline due to the axial symmetry of spin-coating. We report a new method that applies a nonuniform electric field during the spin-coating process. By arranging the field direction to be stationary in the rotating frame, we are able to break the axial symmetry and to orient the colloids along one predefined direction. By regulating the applied field strength, we

demonstrate local control over the orientation of the crystallites, and thus, the orientation is determined by the applied field strength.

\section{INTRODUCTION}

Spin-coating is a widely used technique to produce thin films of polymers. $^{1-6}$ The fluid dynamics of spin-coating has been studied extensively from the perspective of thinning Newtonian liquid droplets ${ }^{1,3,4,6}$ or of particle-laden droplets. ${ }^{7-10}$ The subject still remains a topic of active interest even five decades later after the work of Emslie et al. ${ }^{1}$ due to the complexities involved in studying the fluid flow of non-Newtonian liquids, ${ }^{11}$ as well as the instabilities that occur in the early stages of thinning. ${ }^{12,13}$ As a result, this field continues to attract new methods to study the spin-coating process in detail ${ }^{14,15}$ however, attempts to control external forces during the spincoating process have not previously been explored.

Lately, spin-coating has been extended to colloids. ${ }^{10,14,16-20}$ Much recent work has focused on the use of spin-coating as a robust and reproducible technique to make colloidal

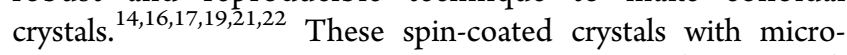
meter-scale building blocks show structural colors (iridescence) when illuminated with white light and display centimeter-scale fourfold or sixfold symmetry that arises from the nature of the microscopic packing of colloidal particles. ${ }^{19}$ These observed symmetries make spin-coating of colloids different from spincoating of polymers; however, the emergence of these symmetries has been studied very little. Interestingly, the symmetric radial arms seen in colloid spin-coating are not an indicator of high crystallinity, and it has been shown ${ }^{14,19,21,22}$ that, due to the axial symmetry imposed by the spin-coating, these crystals are intrinsically polycrystalline with the different crystallites being orientationally correlated with one another.
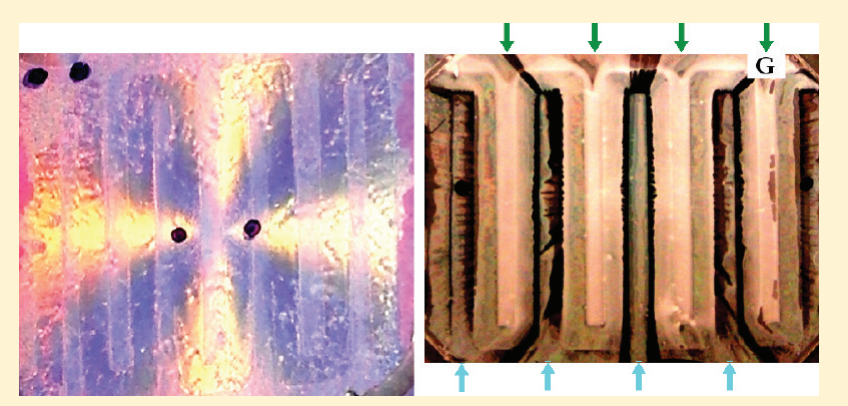
These are hence termed "orientationally correlated polycrystals" (OCP). ${ }^{19}$

Monodomain, defect-free colloidal crystals are of great interest in fabricating inexpensive photonic band gap materials. ${ }^{23-27}$ The intrinsic polycrystallinity of spin-coated colloidal films is thus a major obstacle to realizing viable photonic materials. In other techniques, ${ }^{28-30}$ directional drying is the key to getting large single crystals. Control of crystallite orientation via application of uniform $^{31}$ and nonuniform ${ }^{32}$ electric fields has also been demonstrated. Unfortunately, these techniques lack both the reproducibility of spin-coating and its scalability to larger scale manufacturing methods.

In this work, we demonstrate control in orienting the crystallites by explicitly breaking the axial symmetry of spincoating and picking a prescribed direction. We aim to control the orientation of the crystallites in a specific direction by applying nonuniform external alternating electric fields, which affect the outward flow of fluid during spin-coating. The applied field strength thus regulates the orientation of the crystallites.

\section{EXPERIMENTAL SECTION}

Dispersions of concentration $20 \% \mathrm{v} / \mathrm{v}$ are made with $458 \pm 2 \mathrm{~nm}$ diameter spherical silica particles suspended in methyl propyl ketone (MPK). The detailed procedure in preparing the dispersion is welldescribed in other literature. ${ }^{14,19}$

Received: October 21, 2011

Revised: December 31, 2011

Published: January 7, 2012 


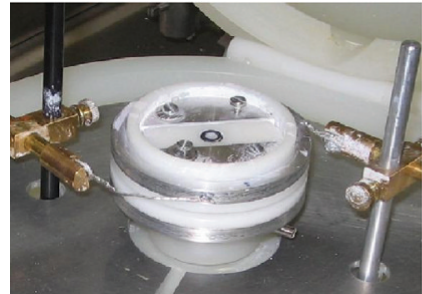

(a)

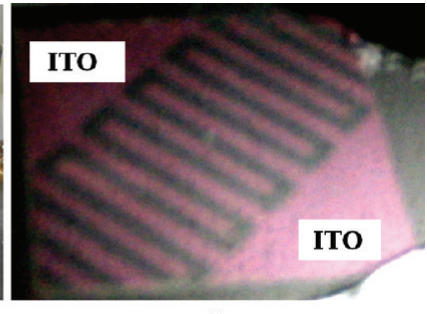

(b)
Figure 1. Photograph of the experimental components. (a) Modified spin-coating chuck (see schematic and construction details provided in Supporting Information). (b) Patterned indium tin oxide (ITO) substrate (size: $20 \times 20 \mathrm{~mm}^{2}$ ).

To apply an electric field whose orientation in the $x-y$ plane rotates with the sample (static in the rotating frame), a custom chuck was constructed, as shown in Figure 1a. This chuck has two electrodes on either side of the spinning center that holds the substrate. Each electrode is connected to one end of a patterned indium tin oxide (ITO) glass substrate-see Figure $1 \mathrm{~b}$. The conducting ITO makes direct electrical contact with the dispersion. One end of the pattern is connected to an alternating high voltage amplifier while the other is grounded, each via a contact made with one of two outer conducting rings on the chuck. Wires connected to the amplifier brush against the outer rings. The amplifier is fed a sinusoidal signal from a function generator. A detailed schematic with construction details is provided in Supporting Information.

Prior to the experiments, the field is turned on and adjusted to desired values. Once the field is set, the substrate is spun at $2000 \mathrm{rpm}$. When the substrate reaches a constant angular velocity, $40 \mu \mathrm{L}$ of the colloidal dispersion is pipetted onto it. After the spun dispersion is completely dry, the spinning is stopped and the field is turned off.

\section{RESULTS AND DISCUSSION}

It has previously been shown that the maximal extent of singlecrystal domains in spin-coated samples is intrinsically limited by the angular spread that results from axial symmetry. ${ }^{19}$ Moreover, the symmetric optical reflections shown in Figure $2 \mathrm{c}$ do not arise from a long-range translational order, but from long-range orientational order. Thus, to obtain long-range translational order, one has to break the axial symmetry, which we achieve in this work via an external alternating electric field.

The colloidal dispersion experiences a nonuniform force when the field is on. The dominant mechanism for this fieldinduced migration of fluid is dielectrophoresis (DEP). At low frequencies, electro-osmotic effects can also play a role.
Detailed frequency dependence is currently being explored; however, as a first approximation, one can identify 3 dielectric media in this system: the colloidal particle (in our case, $\varepsilon_{\mathrm{p}} \sim 4$ ), the solvent $\left(\varepsilon_{\mathrm{s}}=15.5\right)$, and air $\left(\varepsilon_{\mathrm{air}}=1\right)$. One can then write down two Clausius-Mosotti factors $\left(K_{\text {solvent-air }}=0.8\right.$ and $\left.K_{\text {particle-solvent }}=-0.3\right)$. Accordingly, in our experiments, it is expected that solvent-air DEP will be the predominant mechanism, while particle-solvent DEP can additionally be relevant. A demonstration of this dielectrophoretic localization (without spinning) is shown in Figure 2a,b. The colloidal dispersion inside the circle drawn in Figure 2a is redistributed toward the electrode region when a field of $0.47 \mathrm{kV} / \mathrm{mm}$ is turned on (in this particular case a frequency of $40 \mathrm{kHz}$ was employed). Upon drying, it leaves a thick white deposit (Figure $2 \mathrm{~b})$. In the presence of spinning, for the case of zero field, orientationally correlated polycrystals ${ }^{19}$ are obtained (Figure 2c). When the external field is applied, we observe (by eye) two changes in the spin-coated sediment: the deposits of colloidal particles are localized to certain areas of the pattern (Figure $2 \mathrm{~d}$ ), and the reflection arms due to axial symmetry (seen in zero field; Figure 2c) are not obtained.

To study the microscopic orientation of the domains, scanning electron micrographs (SEM) are obtained at different sample positions relative to the center of rotation. The angle between this radial vector and the direction of the pattern in the substrate is termed the "pattern angle" $\alpha$ (Figure 3a). Dotted lines show the directions at which the $\mathrm{OCP}^{19}$ would orient at zero field. In the patterned sample, these positions can fall into one of two regions in the sample (Figure $3 \mathrm{~b}$ ), namely, either the ITO electrode region or the glass nonconducting region between two ITO electrodes.

The dominant orientation of the domains $(\theta)$ in each micrograph is obtained via particle analysis routines that are optimized for crowded particle features in the scanning electron micrographs. ${ }^{33}$ In Figure 4a, $\theta$ is plotted against $\alpha$. At zero field, the orientation of domains is expected to follow $\alpha$, i.e., $\theta=\alpha$. ${ }^{19}$ This is indeed observed in the SEM image in Figure 3c, as well as in the plot in Figure 4a (circles). On applying the field, the axial symmetry is broken and the hydrodynamic shear forces and the electrostatic forces compete in trying to orient the domains (Figure 3d). In the presence of a field, perfect orientation along the field would result in $\theta=0$, independent of $\alpha$. It can be seen in Figure $4 \mathrm{~b}$ that the domain orientation induced by the presence of the field is much more effective in

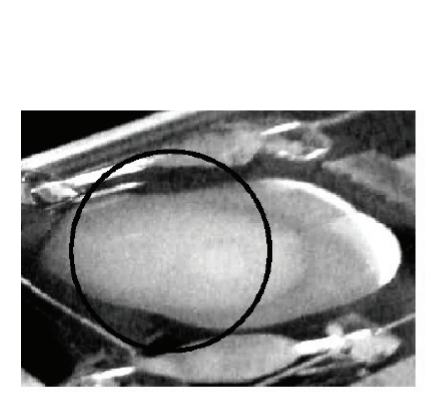

(a)

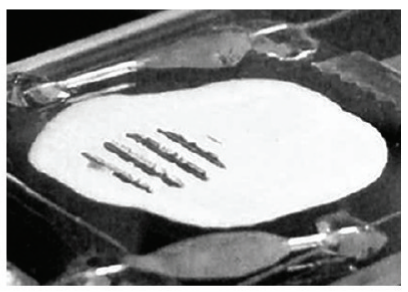

(b)

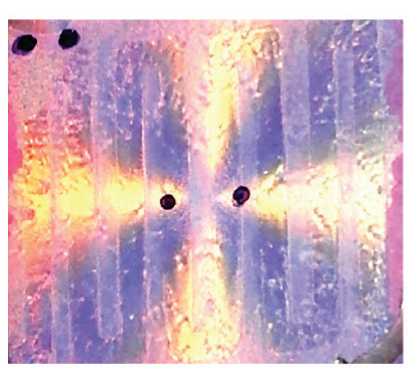

(c)

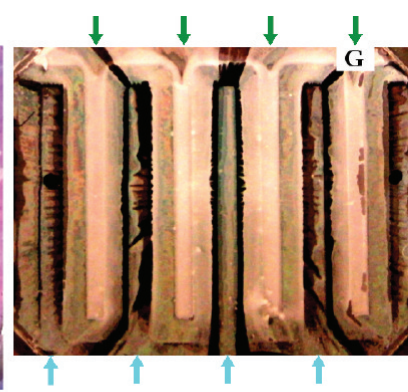

(d)

Figure 2. (a,b) Demonstration of dielectrophoretic localization of colloidal dispersion in the absence of spinning. (a) In the absence of a field, the white colloidal suspension is clearly visible. (b) With field, $0.47 \mathrm{kV} / \mathrm{mm}, 40 \mathrm{kHz}$. Here, the colloidal suspension has been redistributed toward the electrode regions. (c) Spin-coated sample at zero field shows four-arm patterns characteristic of axisymmetric spin-coating. (d) Spin-coated sample with field, $0.95 \mathrm{kV} / \mathrm{mm}, 3 \mathrm{kHz}$. Green and blue arrows show the interdigitated ITO electrodes connected to the amplifier (green arrows point to the grounded electrode G). Here, the final sediment shows no remnant of axial symmetry. 


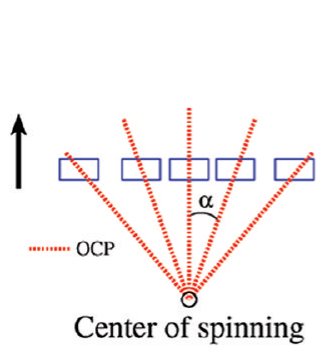

(a)

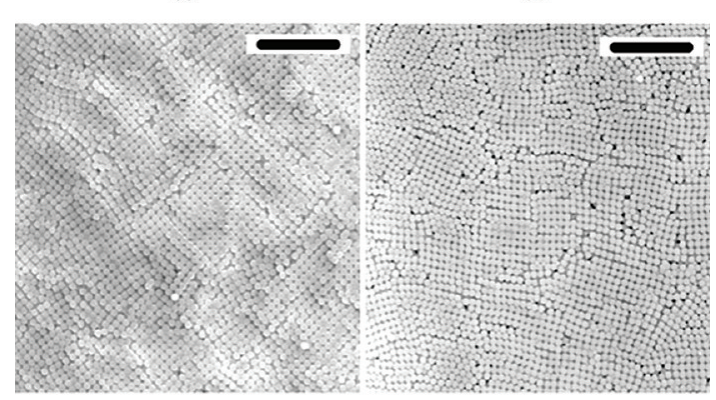

(c)

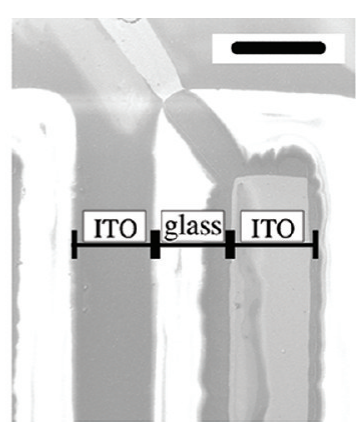

(b)

(d)
Figure 3. (a) Sketch for the positions of the analyzed SEM micrographs (rectangles). $\alpha$ is the "pattern angle", defined by the angle between the direction of the pattern (arrow shown) and the radial position of the image relative to the center of rotation. Dotted lines illustrate the directions at which the orientationally correlated polycrystals would be oriented at zero field. (b) Low magnification SEM overview of relevant regions in the samples (glass and ITO). Scale bar is $1 \mathrm{~mm}$. (c) SEM micrograph for the zero field condition, $\alpha$ $=45^{\circ}$. (d) SEM micrograph in the glass region $(0.95 \mathrm{kV} / \mathrm{mm}$ at 3 $\mathrm{kHz}$ ), $\alpha=49^{\circ}$. Scale bars (for $\mathrm{c}$ and $\mathrm{d}$ ) are $5 \mu \mathrm{m}$.

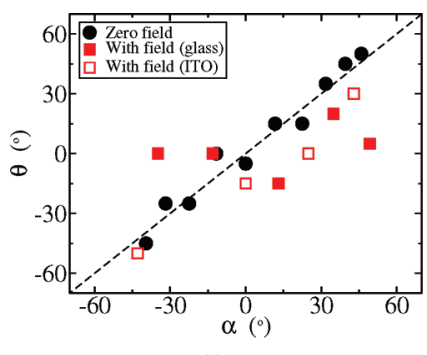

(a)

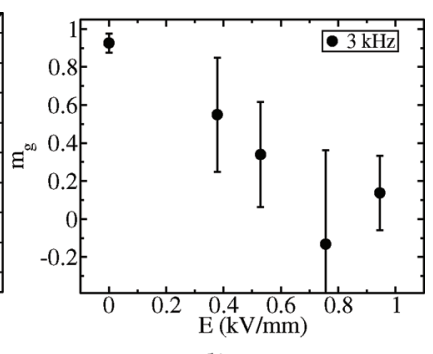

(b)
Figure 4. (a) Dominant domain orientation angle $\theta$ as a function of $\alpha$. For clarity, the zero-field case (circles) and only one case with applied field $(0.95 \mathrm{kV} / \mathrm{mm}$ at $3 \mathrm{kHz}$; squares) are plotted. Open and filled squares represent the ITO and the glass region on the substrate, respectively. The condition for orientationally correlated polycrystals $(\theta=\alpha)$ is shown by the dashed line. Domain orientation along the ITO pattern corresponds to $\theta=0$. (b) Slopes of the linear fits, $m_{g}$, for the dominant orientation of domains (glass region data) against the applied field strength show a systematic decrease from close to unity (OCP) to close to zero (direction of the ITO pattern).

the glass regions of the substrate where the field gradient is higher than in the ITO regions-a map of the simulated electric potential is included in Supporting Information Figure 2.

To characterize the effect of the applied field, we linearly fit the dominant orientations, $\theta$, for the glass regions. The slopes of these fits, $m_{g}=\mathrm{d} \theta / \mathrm{d} \alpha$, give quantitative information on the efficacy of the field in orienting the domains. Unidirectional orientation of the colloidal crystalline domains along the pattern corresponds to $\theta=0$. Thus, when the field fully determines domain orientation, $\theta=0$, independent of $\alpha$, and $m_{g}$ $=0$. Figure $4 \mathrm{~b}$ shows the slope $m_{g}$ as a function of field strength. In this plot, only data points from the glass region are included. The slope $m_{g}$ shows a marked decrease from a value of 1 , which is expected for an OCP structure, to a value close to zero, which is expected for field-induced orientational order.

On the other hand, the calculated slope for the ITO regions is closer to unity (data not shown). An asymmetry is also observed in the colloidal deposit between the ground and oscillating voltage electrode regions (Figure $3 \mathrm{~b}$ ). This could possibly be related to opposing solvent-in-air and particles-insolvent dielectrophoretic flows and/or to some capacitive effects in the system. Further experiments are in progress to study this phenomenon.

\section{CONCLUSIONS}

Many strategies have been explored to fabricate periodic arrays of nanoparticles and microparticles. ${ }^{34,35}$ These include colloidal sedimentation, ${ }^{31,36,37}$ controlled drying, ${ }^{28-30}$ and external force fields. ${ }^{32,38,39}$ Spin-coating of colloids, at least at present, produces crystals of lower quality than the above techniques; however, the robustness and reproducibility of spin-coating (which is already established commercially to make polymer thin films), as well as its scalability toward manufacturing processes, makes it very attractive to explore strategies for optimizing and improving the quality of the crystals obtained from the spin-coating process.

We have built a device that allows the application of an electric field in the rotating frame of a commercial spin-coater. An applied electric field during the spin-coating of colloids breaks axial symmetry and forces a preferred orientation on the resulting colloidal crystal over millimeter-scale distances. We show that a plausible mechanism for this change in orientational order is that the applied alternating electric field during the spin-coating process affects hydrodynamic flows via dielectrophoretic confinement of the suspension, utilizing the suspension-air dielectric contrast. The fact that the flows are driven by suspension-air dielectric mismatch implies that this technique could also be viable for patterning polymer films. Moreover, unlike particle DEP techniques where the threshold fields scale inversely with particle volume, the methods reported here are scalable to nanocolloids.

While field-assisted spin-coating removes the fundamental constraint in obtaining long-range translational order, singlecrystalline domains of large extent are the next challenge. This will require fine-tuning of the electrode spacing length scales. The technique has not been optimized with respect to another parameter, the solvent evaporation rate. The slower the evaporation rate, the better the domain orientation will be. In addition, a recent report of stroboscopic imaging of structure during spin-coating ${ }^{15}$ might afford a means to study the fieldinduced self-organization in greater detail. Further experiments will focus on these issues.

\section{ASSOCIATED CONTENT}

\section{Supporting Information}

Additional information as described in the text. This material is available free of charge via the Internet at http://pubs.acs.org. 


\section{AUTHOR INFORMATION}

\section{Corresponding Author}

*E-mail: ayethiraj@mun.ca.

\section{Present Addresses}

"Department of Physics and Astronomy, McMaster University, Hamilton, Canada

$\square$ Department of Physics, University of Guelph, Guelph, Canada

\section{Author Contributions}

${ }^{\mathrm{I}} \mathrm{APB}$ and MP contributed equally to this work.

\section{ACKNOWLEDGMENTS}

We acknowledge Edward Hayden and Andrew R. Morrow for their assistance during the experiments, and Claire $\mathrm{F}$. Woodworth for carefully reading the manuscript in order to correct grammatical errors. This work is partly supported by NSERC and the Spanish Government Contract No. FIS200801126. M.G. and M.P. acknowledge financial support from the "Asociación de Amigos de la Universidad de Navarra".

\section{REFERENCES}

(1) Emslie, A. G.; Bonner, F. T.; Peck, L. G. J. Appl. Phys. 1958, 29, 858.

(2) Heriot, S. Y.; Jones, R. A. L. Nat. Mater. 2005, 4, 782.

(3) Meyerhofer, D. J. Appl. Phys. 1978, 49, 3993.

(4) Birnie, D. P. III; Manley, M. Phys. Fluids 1997, 9, 870.

(5) Münch, A.; Please, C. P.; Wagner, B. Phys. Fluids 2011, 23, 102101.

(6) Cregan, V.; O’Brien, S. J. Colloid Interface Sci. 2007, 314, 324.

(7) Cook, B. P.; Bertozzi, A. L.; Hosoi, A. E. SIAM J. Appl. Math. 2008, 68, 760

(8) Frastia, L.; Archer, A. J.; Thiele, U. Phys. Rev. Lett. 2011, 106, 077801.

(9) Warner, M. R. E.; Craster, R. V.; Matar, O. K. J. Colloid Interface Sci. 2003, 267, 92.

(10) Rehg, T.; Higgins, B. AIChe J. 1992, 38, 489.

(11) Acrivos, A.; Shah, M. J.; Petersen, E. E. J. Appl. Phys. 1960, 31, 963.

(12) Fraysse, N.; Homsy, G. M. Phys. Fluids 1994, 6, 1491.

(13) Melo, F.; Joanny, J. F.; Fauve, S. Phys. Rev. Lett. 1989, 63, 1958.

(14) Giuliani, M.; González-Viñas, W.; Poduska, K. M.; Yethiraj, A. J. Phys. Chem. Lett. 2010, 1, 1481.

(15) Ebbens, S.; Hodgkinson, R.; Parnell, A. J.; Dunbar, A.; Martin, S. J.; Topham, P. D.; Clarke, N.; Howse, J. R. ACS Nano 2011, 5, 51245131

(16) Jiang, P.; McFarland, M. J. Am. Chem. Soc. 2004, 126, 13778.

(17) Mihi, A.; Ocaña, M.; Míguez, H. Adv. Mater. 2006, 18, 22442249.

(18) Shereda, L.; Larson, R.; Solomon, M. Phys. Rev. Lett. 2008, 101, 038301.

(19) Arcos, C.; Kumar, K.; González-Viñas, W.; Sirera, R.; Poduska, K.; Yethiraj, A. Phys. Rev. E 2008, 77, 050402R.

(20) Pichumani, M.; González-Viñas, W. Magnetohydrodynamics 2011, 47, 191.

(21) Wu, Y. L. Control over colloidal crystallization by shear and electric fields. Ph.D. thesis, Utrecht University, 2007.

(22) Vermolen, E. Manipulation of Colloidal Crystallization. Ph.D. thesis, Utrecht University, 2008.

(23) Braun, P. V.; Wiltzius, P. Nature 1999, 402, 603.

(24) Velev, O. D.; Kaler, E. W. Adv. Mater. 2000, 12, 531.

(25) Joannopoulos, D. Nature 2001, 414, 257.

(26) Norris, D. J.; Vlasov, Y. A. Adv. Mater. 2001, 13, 371.

(27) Vlasov, Y. A.; Bo, X. Z.; Sturm, J. C.; Norris, D. J. Nature 2001, 414, 289.

(28) Denkov, N. D.; Velev, O. D.; Kralchevsky, P. A.; Ivanov, I. B.; Yoshimura, H.; Nagayama, K. Langmuir 1992, 8, 3183.
(29) Jiang, P.; Bertone, J. F.; Hwang, K. S.; Colvin, V. L. Chem. Mater. 1999, 11, 2132.

(30) Gu, Z. Z.; Fujishima, A.; Sato, O. Chem. Mater. 2002, 14, 760.

(31) Yethiraj, A.; Thijssen, J. H. J.; Wouterse, A.; van Blaaderen, A. Adv. Mater. 2004, 16, 596.

(32) Juárez, J.; Bevan, M. J. Chem. Phys. 2009, 131, 134704.

(33) Macdonald, M.; Yethiraj, A.; Beaulieu, L. (Submitted) 2011.

(34) Velev, O. D.; Gupta, S. Adv. Mater. 2009, 21, 1897.

(35) Shevchenko, E. V.; Talapin, D. V.; Kotov, N. A.; O’Brien, S.; Murray, C. B. Nature 2006, 439, 55.

(36) Hoogenboom, J. P.; Yethiraj, A.; van Langen-Suurling, A. K.; Romijn, J.; van Blaaderen, A. Phys. Rev. Lett. 2002, 89, 256104.

(37) Hoogenboom, J. P.; Vergeer, P.; van Blaaderen, A. J. Chem. Phys. 2003, 119, 3371

(38) Yethiraj, A.; van Blaaderen, A. Nature 2003, 21, 513.

(39) Xie, R.; Liu, X.-Y. Adv. Funct. Mater. 2008, 18, 802. 